\title{
Simulating Fire Effects on Complex Building Structures
}

\author{
HOWARD R. BAUM \\ Building and Fire Research Laboratory \\ National Institute of Standards and Technology (NIST) \\ Gaithersburg, Maryland, 20899, USA
}

\begin{abstract}
The purpose of this lecture is to assess the current state of our ability to simulate the consequences of a fire in a large building, and suggest some areas where improvement is needed. Attention is focused on the coupling of fire dynamics simulations and heat transfer analyses to each other and to structural analyses of the damaged building. The role that uncertainty in "input parameters" resulting from coupling a sequence of complex simulations is considered. The methodology used in the NIST investigation into the collapse of the World Trade Center Towers will be described from this perspective. The intent is not to summarize the results of the investigation, but rather to provide a specific context that illustrates the strengths and weaknesses of the methodologies employed. Research needs are emphasized by examination of some basic problems in fire-structure interactions.
\end{abstract}

KEY WORDS: fire modeling, heat transfer, fire-structure interactions

\section{INTRODUCTION}

There has been a resurgence of interest in the response of building structures to fires over the past several years. This interest was greatly enhanced by the attack on, and subsequent collapse of, the World Trade Center (WTC) towers. This has resulted in at least three detailed physics-based analyses of the collapse of the towers in the literature. Usmani [1] and Hori [2] concentrated on the degradation of the towers given assumed temperature distributions in the load bearing structures. Abboud et al. [3] performed a more comprehensive analysis, dealing with both the initial impact of the aircraft as well as the final collapse, using as much photographic and video evidence as was available at the time [4], [5]. More studies are surely on the way. NIST has just completed its own investigation, the details of which will be reported elsewhere.

Rather than debate the causes of the collapse of the towers, it seems more useful at present to discuss the methodology used to study the collapse. Many if not most of the issues that had to be addressed in the WTC simulations would arise in the analysis of any major fire affecting the structural stability of a large building. The complexity of such buildings, together with the need to couple phenomena usually considered in isolation, forces compromises in methodology not required for simpler fire scenarios. This statement certainly applies to the analyses cited above, as well as the NIST investigation. Hopefully, the discussion will shed some new light on our ability to simulate such events.

First, the sequence of simulations of the consequences of the attacks on the towers and the software employed are discussed briefly. Very few details will be given, as the purpose 
is primarily to orient the reader. Since the software employed is widely available either as commercial products or NIST freeware, it is fair to assume it is representative of the current state of the art (indeed, it was chosen primarily for this reason). This is followed by a discussion of the approximations needed to couple the various elements of the simulation together. The role of fire-induced uncertainty and the extent to which the descriptions of the different physical processes must be coupled will be emphasized. A sampling of results (drawn primarily from the cited references) will be used to illustrate these points. The paper will conclude with some recommendations for future research.

\section{THE NIST APPROACH - A SYNOPSIS}

The NIST-led analysis of the events initiated by the attack on the towers is composed of four parts; the initial impact, the fire dynamics, thermal analysis of the load-bearing structure, and structural deterioration. The information flow in principle proceeds as follows: The initial impact simulation is needed as input into the following three parts. It partially defines the geometry used for the fire dynamics simulation, provides guidance about insulation damage that affects the heat transfer calculation, and determines the state of the structure that survived the impact. The fire dynamics provides the thermal environment in the gas phase needed to determine the heat transfer to the exposed building surfaces. The thermal analysis determines the temperature distribution in the load bearing structure. Finally, the structural analysis integrates all the previous information to make predictions about the loads and deflections up to the point of collapse. The structural dynamics during the final collapse of the towers is not part of the investigation.

\section{Impact Analysis}

Simulation of the initial impact of the aircraft on the towers, performed under contract, covers approximately the first 0.6 seconds of elapsed time. The simulations were performed using the commercial code LS-DYNA, "a general purpose finite element code for analyzing the large deformation dynamic response of structures including structures coupled to fluids" [6]. It should be noted that although the code has some fluid mechanics simulation capability, it cannot simulate combustion phenomena. Thus, no attempt was made to simulate the initiation of the fireball in this part of the investigation. LS-DYNA and the finite element code FLEX [7] used by Abboud et al. share a common ancestor DYNA-3D, although both are undoubtedly much more capable now. The codes both used highly detailed representations of the aircraft structure and those floors of the WTC towers in the immediate vicinity of the impact to produce an estimate of the damage inflicted on the load bearing structure by the breakup of the aircraft.

The predicted damage to the exterior of the towers was verified by direct comparison with the highly detailed photographic and video evidence. The initial configuration, material properties, and relative velocity of both aircraft and building are known quite accurately. The level of sophistication in the computer codes then permits an impressively detailed prediction of the breach of the outer perimeter of the towers. However, the most important outputs of the analysis are the prediction of the post-impact interior geometry and the degree of damage to the insulation. For the purposes of the present paper, it completes the description of building needed for subsequent analyses. Thus, the remainder of this work will regard the post-impact state of the building as defined by these calculations as 


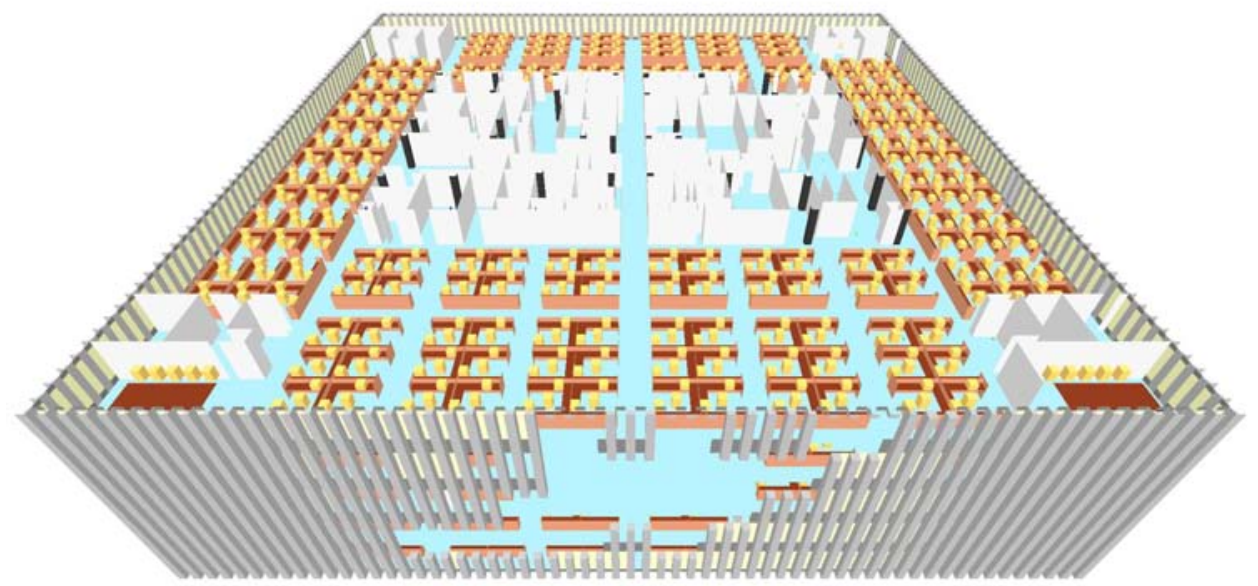

Fig. 1. Layout of North WTC tower model as used in FDS simulations. Windows, interior partitions, and workstation geometry are all included. However, the columns are not to scale and no internal floor system structural components are present in the simulations. Courtesy K. McGrattan [8].

the initial conditions for the fire dynamics, thermal, and structural analyses.

\section{Fire Dynamics}

The fires induced by the aircraft impact were studied using the NIST Fire Dynamics Simulator (FDS), a finite difference based computational fluid dynamics code solving the low Mach number form of the Navier-Stokes equations appropriate for combustion, smoke and heat transport generated by fires [8]. FDS was modified to enable parallel processing as part of this study. [9]. The code uses a geometry model of those floors of the towers affected by fires. The geometry model differs substantially from that used for the impact analysis, since it is based on architectural rather than structural features. The spatial resolution employed is too coarse to include much of the load-bearing structure, retaining only the floor slabs. The core and exterior columns are present but cannot be represented to scale. On the other hand, it includes the office partitions and work stations. The model also includes vents opened up either by estimates of damage caused by the initial impact or windows broken during the fire. Figure 1 shows the geometry used by FDS. Every feature shown in the figure is included in the simulations. No attempt was made to predict window breaking. Photographic evidence was used to obtain times and locations of window breakage and the resulting information was specified as part of the input boundary conditions.

The fireballs resulting from the initial impact were studied separately to estimate the fuel consumed outside the towers and thus unavailable as an accelerant. Two independent techniques were used to obtain estimates of both the fuel consumption and the uncertainty of the estimate. FDS simulations were performed by Rehm et al. [10] based on a variety of assumptions about the internal configuration of the towers following impact. Baum and 
Rehm [11] performed an analytical study assuming the shape of the fireballs emerging from each face of the towers to be hemispherical. Comparison of these calculations with video images yielded estimates that ten to twenty-five percent of the aircraft fuel was consumed.

The FDS simulations of the fires for the remaining life of the towers were tested by comparing predicted temperatures and heat release rates with those measured in a series of laboratory experiments. These experiments burned paper laden work stations similar to those used in the WTC towers in a compartment with limited openings resembling those in the towers. The actual office contents, their condition following the impact, and the completeness of burning are additional sources of uncertainty. Many simulations of the actual fires in the towers with varied assumptions about the input parameters were performed to test the sensitivity of the model. Calculated flame locations near the perimeter were compared with photographic and video images. Details of these and other aspects of the WTC fire simulations can be found in McGrattan and Bouldin [9].

\section{Thermal Analysis}

The thermal analysis of the structure couples the fire dynamics to the calculations of stress and deflection that ultimately determine the collapse path of the towers. The analysis itself is composed of two parts; the calculation of the local heat transfer from the heated gases to the exposed surfaces of insulation materials and structural components, and the heat flow through the condensed phase materials. A decision was made to use the code ANSYS [12] to perform the structural analysis. Since ANSYS also has a thermal analysis capability, this led the development of a methodology that couples ANSYS to FDS. This methodology, the Fire Structure Interface (FSI), is capable of generalization to other codes and other structural fire problems. It makes use of a mixture of analytical and computational techniques to cope with the wide disparity of length and time scales that control the physical processes simulated in FDS and whatever structural analysis code is chosen for use. A more detailed description of the coupling procedures that comprise FSI is presented in the next section. These techniques are also summarized in Prasad and Baum [13].

The finite element model used for the ANSYS simulation of the heating of the structure is the most detailed of all those used for the investigation. It is composed entirely of threedimensional "brick" elements, and represents all the beams, columns, and floor systems on all floors affected by the fires (including the floors immediately above and below floors on which fires occured). It incorporates detailed models of the protective insulation, including estimates of damage obtained from calculated debris patterns performed as part of the impact analysis. Random irregularities in the thickness of spray-on insulation are also taken into account. Figure 2 shows the perimeter columns and connections as simulated for the thermal analysis. The floor system and core columns are shown in Fig. 3. Analogous models were made for the South tower. The accuracy of the calculations was tested by comparing FSI predictions with a series of large scale experiments on steel trusses and columns. Studies of this type have been performed by Hasemi and his collaborators [14]. The results indicate that FSI can reliably predict the temperature in structural components and that it does not add any more uncertainty to the overall analysis than that already present in the FDS simulations. A detailed analysis of the experimental uncertainty and model sensitivity associated with both the fire dynamics and thermal analysis of the laboratory experiments is presented elsewhere in this symposium [15]. 


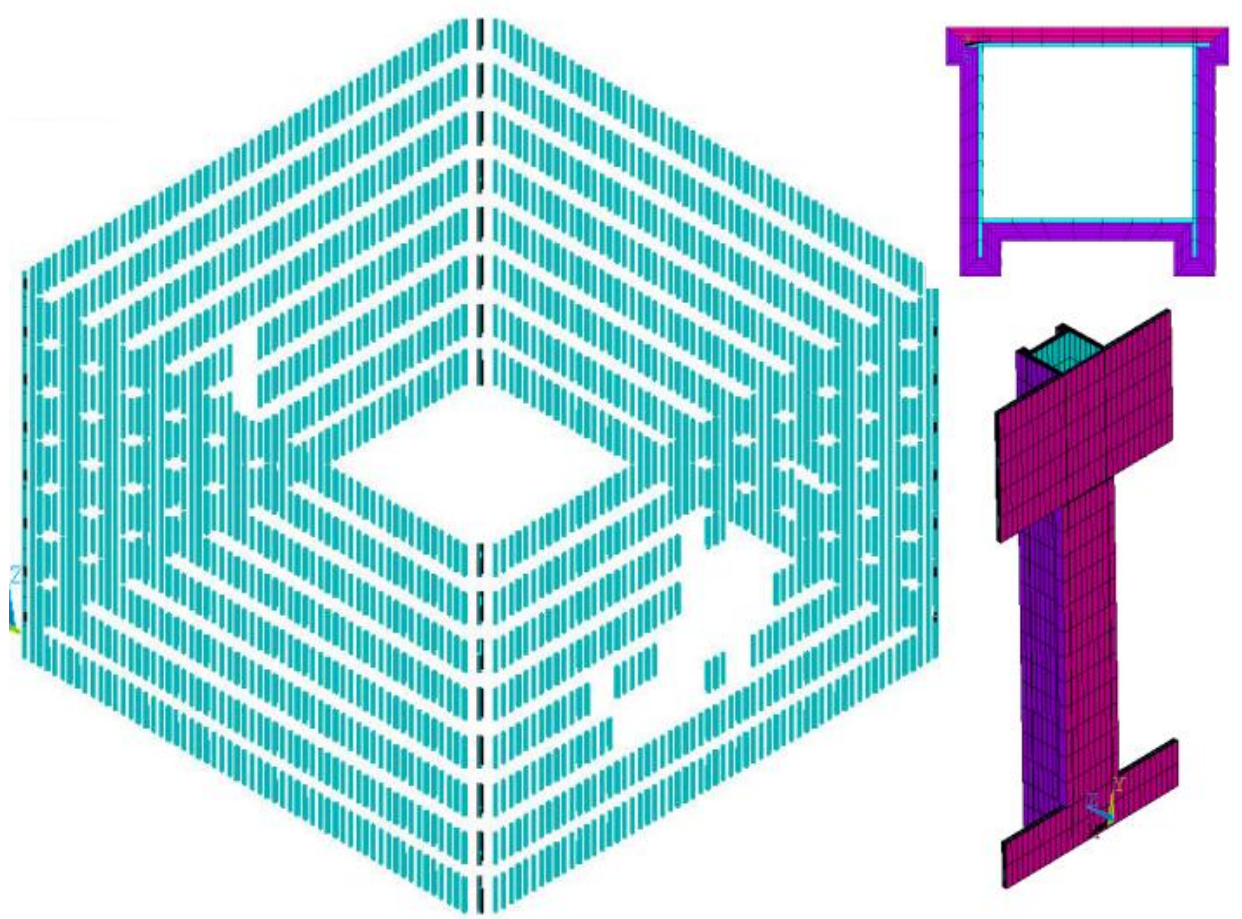

Fig. 2. The perimeter column structure of North WTC tower shown as simulated in the thermal analysis using ANSYS. The details of the column shapes and connection with spandrels are shown as inserts. Courtesy K. Prasad.

This level of detail is necessary precisely because the calculations it supports provide the transition from large scale convective and radiative transport in the gas to highly localized thermal conduction through solids. It is also needed because it turns out that the temperature distributions in the steel are more sensitive to the fireproofing thickness and the degree of impact damage to the insulation than any other quantity. It is feasible only because heat transfer by thermal conduction (even non-linear conduction with non-linear radiative boundary conditions) is much simpler computationally than either turbulent combustion or non-linear solid mechanics.

\section{Structural Analysis}

The stresses and displacements in the structure were calculated using ANSYS by a contractor with the thermal input from FSI and the initial impact damage estimate obtained from LS-DYNA. A series of highly detailed component models of relevant parts of the structure were subjected to a variety of loads. These were used to develop a less detailed set of floor-level models as well as multi-floor models of portions of the perimeter column system. Video images were used to select critical portions of the perimeter columns for detailed analysis. Finally, a global model based on simplifications derived from sensitivity 


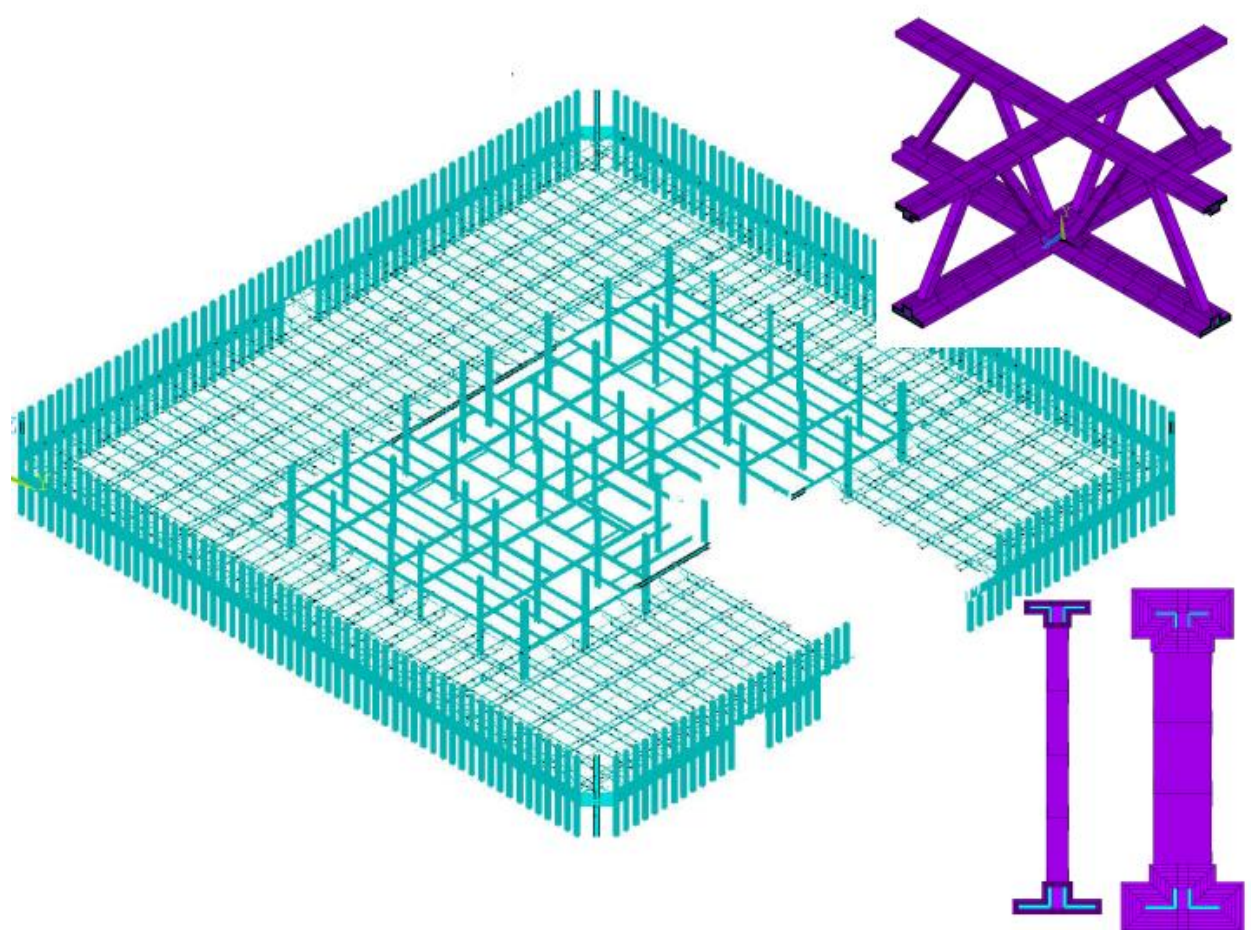

Fig. 3. The North WTC tower floor system (with concrete slab removed for clarity) and core columns shown as simulated in the thermal analysis using ANSYS. The details of the trusses including the insulation are shown as inserts. Additional illustrations can be found in [13]. Courtesy K. Prasad.

analyses using the component and floor level models was developed. It should be noted that local models and/or highly simplified floor system models were also used by Usmani [1] and Hori [2] in their analyses.

An interpolation scheme (part of FSI) was used to input the predicted temperatures into the finite element models employed for the structural analysis. The thermal information was transferred to the ANSYS structural model at intervals of ten minutes of simulated time for each of the towers. Neither Usmani nor Hori attempted this step. This process introduces additional uncertainty into those calculations using the less detailed structural models, since it represents a loss of information calculated with FSI. However, it is crucial if a given fire scenario is to be associated with structural collapse. These issues will be discussed in further detail below.

\section{COUPLING AND FEEDBACK}

The sections above outline how the overall analysis was broken down and indicate the methods used to study each portion of the overall simulation as an isolated entity. However, if the effects of a given fire on a structure are to be predicted, it is necessary to couple these codes in an internally consistent manner. Since the aircraft impact is both peculiar 
to the WTC attack and considers less than one second of elapsed time, attention is focused on the interactions between the fire dynamics, heat transfer, and structural analysis. These processes occur simultaneously in reality, but are considered sequentially in the WTC analyses. This is a reflection of the current state of the art. I can find no published example of a fully coupled simulation of a fire in a complex structure that includes fire dynamics, heat transfer, and structural mechanics.

At many points in the WTC analyses, photographic and visual evidence are used to compensate for our current inability to perform a fully coupled simulation. The most obvious of these is the effect of window breaking on fire dynamics. While glass windows are not structural components, the "collapse analysis" of such a window requires a coupling of fire dynamics, heat transfer and stress analysis analogous to that needed for the load bearing structure. Fortunately, there is no need to consider this problem in detail since it was the subject of a superb recent review by Pagni [16]. From the present perspective, the most important conclusion to emerge from this review is the following: While the time to initial crack formation in the glass can be reliably estimated as a function of window geometry, materials, and thermal load, the time at which the glass falls out of its frame cannot be predicted with any confidence. Since the fire ventilation will not change until the window pane is removed from its frame, this aspect of the feedback from changes in the building structure to the fire can not yet be quantified.

\section{The Fire Structure Interface}

The coupling between the fire dynamics and the structure is dominated by the radiation heat transfer. The radiation field must be determined from solutions of the radiative transport equation, which relates the incident flux to the spatial distribution of temperature and combustion products (most particularly the distribution of soot particulate) as well as the enclosure geometry. Such calculations are typically performed as part of a CFD based simulation of the fire dynamics. However, the ability to couple such codes as the NIST Fire Dynamics Simulator (FDS) [8] directly to a suitable structural analysis code does not yet exist. The enormous differences in spatial and temporal length scales, differences in numerical techniques, and the complexity of the computer codes makes the development of an efficient coupled analysis of fire-structure interactions a daunting task. This disparity in length scales is illustrated in Fig. 4, which shows a small portion of a WTC floor gridded at the $50 \mathrm{~cm}$ resolution used for the FDS simulations together with a small segment of the floor truss system and an insulated perimeter column as represented in ANSYS. Since the truss rods have a $2.5 \mathrm{~cm}$ diameter and the perimeter columns are $35 \mathrm{~cm}$ on a side it is clear that FDS must be supplemented by a method that permits the heat transfer to the structure to be calculated.

The FSI offers an approach to the calculation of the coupled heat transfer problem. It takes advantage of the fact that the simplest compartment fire models, the "zone models", assume that the compartment is divided into a hot, soot laden upper layer and a cool, relatively clear lower layer. For the purposes of the FSI, the temperature gradients in the horizontal directions are much smaller than those in the vertical direction. The layer temperatures and radiative properties are taken from suitably chosen temporal and spatial averages of output generated by FDS. The time averages are chosen to be compatible with the time scales associated with thermal diffusion through the smallest structural members of interest. 

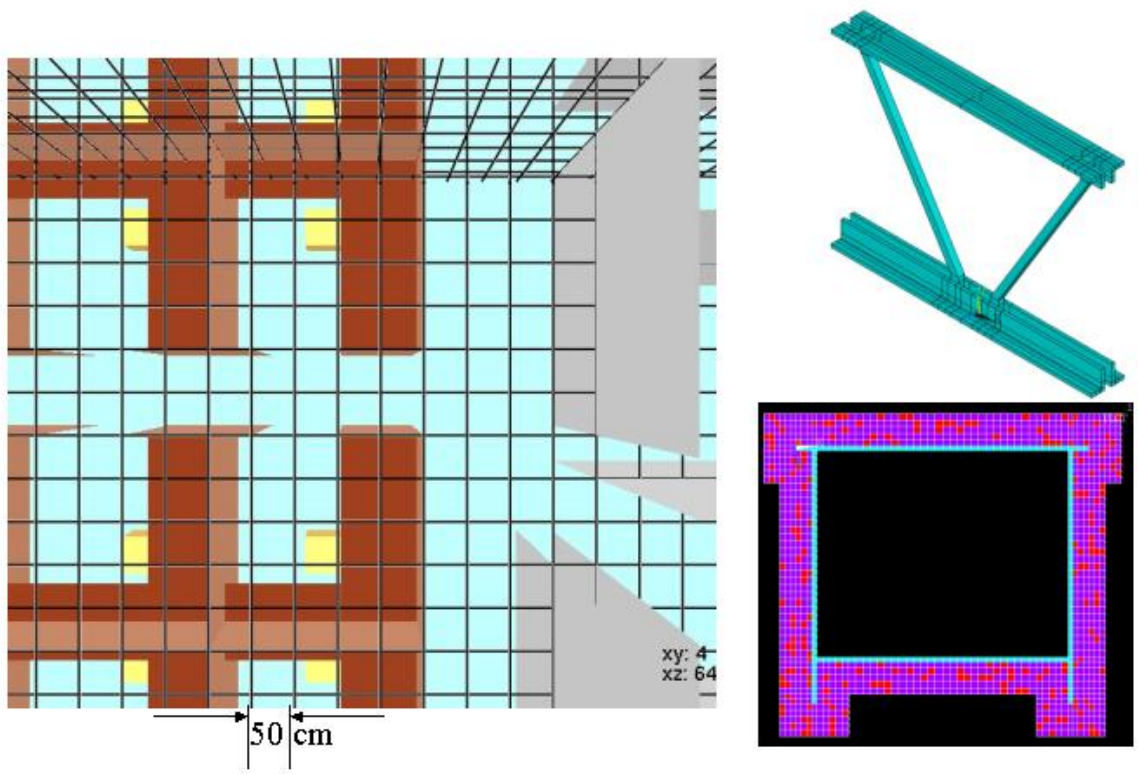

Fig. 4. Portion of WTC floor gridded at resolution used for FDS simulations. A section of the floor truss system and a perimeter column cross-section as modeled in ANSYS are also shown. The truss rods are $2.5 \mathrm{~cm}$ in diameter and the column is $35 \mathrm{~cm}$ on a side. Courtesy K. Prasad.

The spatial averages replace the detailed vertical temperature and absorption coefficient profiles with an effective "zone model" profile. This last approximation is not crucial to the development of the FSI, but is used to reduce the information transfer required between FDS and ANSYS.

The step that is crucial is recognition of the implications of the layered thermal and optical properties of the gas phase. Let length scales in the horizontal directions $\vec{x}$ be scaled with a length $L$ (over sixty meters in the WTC towers), while the grey gas absorption coefficient $\kappa$ and length scales in the vertical direction $z$ are scaled with $H$, the height of an individual floor (less than four meters in the WTC towers). Finally, let the integrated intensity $I\left(\vec{x}, z, \vec{\Omega}_{x}, \Omega_{z}\right)$ be normalized with respect to $\sigma T_{r}^{4}$, where $\sigma$ is the Stefan-Boltzmann constant, $\vec{\Omega}=\left(\vec{\Omega}_{x}, \Omega_{z}\right)$ denotes the local direction of the radiation field, and $T_{r}$ a suitable reference temperature. Then, denoting dimensionless quantities with a tilde, the radiative transport equation takes the form:

$H / L\left(\vec{\Omega}_{x} \cdot \nabla_{x} \tilde{I}\right)+\Omega_{z} \frac{\partial \tilde{I}}{\partial z}=\tilde{\kappa}\left(\frac{(\tilde{T})^{4}}{\pi}-\tilde{I}\right)$

Since the ratio $H / L \ll 1$, the terms in round brackets on the left hand side of Eq. (1) can be ignored. The remaining terms are those associated with the problem of radiative 


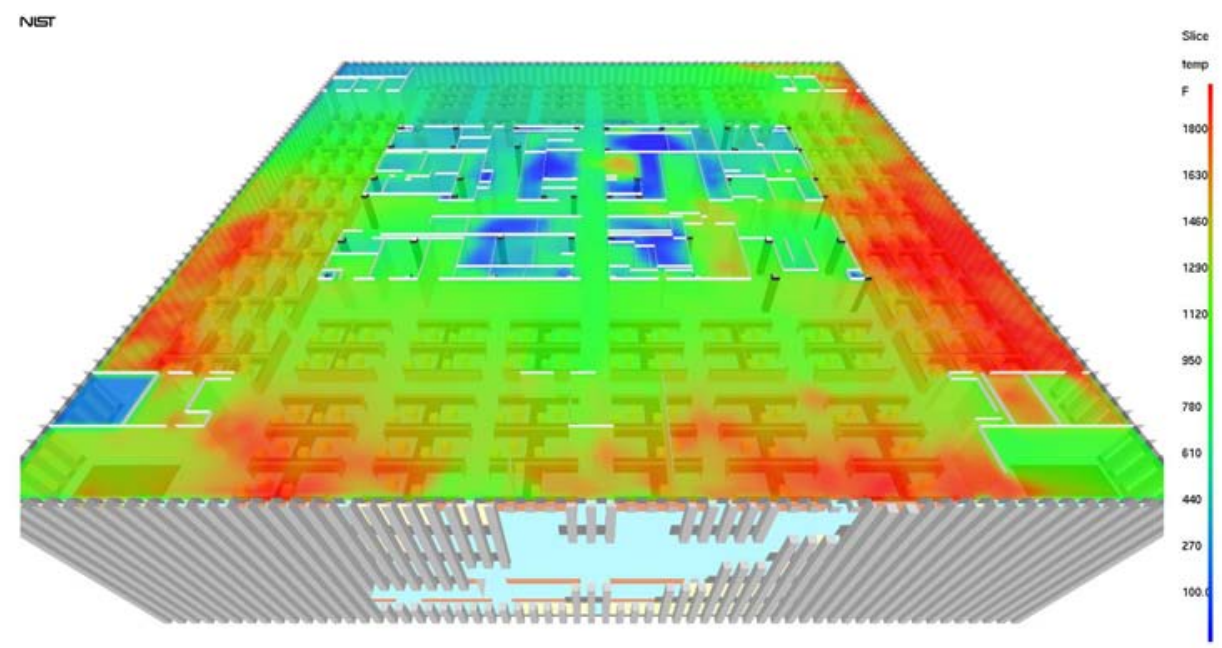

Time: 1000.0

Fig. 5. Predicted upper layer temperature of a floor of the north tower 30 minutes after impact using the model shown in Fig. 1. The peak temperatures are in the vicinity of $1100^{\circ}$ C. Courtesy K. McGrattan [8].

transport between plane parallel layers. For this simplified geometry, the radiative transport equation can be solved exactly and explicit formulae for the heat flux obtained as functions of the temperatures, hot layer depth, soot concentration, as well as the location and orientation of the structural element. The basic analysis is quite well known in the heat transfer community [17], and the extensions needed for use in FSI are readily obtained. Details can be found in [13]. The FSI converts these formulae into input for ANSYS or any other finite element computer code that can be used for both time dependent three dimensional heat transfer within the solid phase and for the stress analysis of the heated structure. The solid phase heat transfer is dominated by thermal conduction with thermal radiation boundary conditions. The input radiation is calculated by FSI based on averages extracted from FDS, while the outgoing radiation is a function of the surface temperatures predicted by ANSYS. A very detailed geometry model of all solids can be accommodated, with solutions that account for insulation damage as well as statistical variations in the application of spray-on fireproofing. Finally, the FSI interpolates the temperatures obtained from this calculation into the geometry model employed for the stress analysis. The complexity of the structural mechanics simulations makes this last step necessary.

\section{Sample simulations}

As noted above, the simulations are performed sequentially, with the FDS simulation coming first. Figure 5 shows a snapshot of the upper layer temperatures in the north tower 30 minutes after impact [8]. This was one of a series of development simulations, and is not necessarily the best prediction of the state of the fires at that time. However, it gives a gen- 
eral idea of the kind of predictions that can be made using this methodology. Note that the fire dynamics on all floors must be computed simultaneously, since the floors are connected by both ventilation shafts and impact damage. The results show fires that slowly migrate around the floor, with peak temperatures in the upper layer in the vicinity of $1100^{\circ} \mathrm{C}$. Figure 5 also shows the relatively smooth temperature variation over most of the upper layer with rapid transitions confined to the edges of the high temperature zones where burning is taking place. This helps justify the plane layer radiative transport analysis used by the FSI.

The computational mesh, even at $50 \mathrm{~cm}$ resolution, requires nearly 150,000 cells per floor. Over 500,000 time steps are needed to advance the calculation for 6000 seconds of simulated time. Without the use of the parallel processing technique incorporated into FDS as part of the WTC investigation, these calculations would be nearly impossible to perform. Indeed, McGrattan and Bouldin [8] estimate that the computation illustrated in figure 5 would take up to two months on a single processor, if it were capable of addressing the 6-12 gigabytes of memory required to perform the computation. This memory requirement would rule out the use of all 32 bit "commodity" processors. The use of parallel processing has reduced the time needed to complete a simulation to as little as two days using eight processors per floor. Moreover, by breaking up the calculation so that the computational demands on each processor are not too large, inexpensive commodity processors in "Beowulf Clusters" connected with gigabit networks can be employed.

Since the time step used by FDS is of order $0.01 \mathrm{~s}$, and the smallest time scales of interest in the thermal analysis are several seconds, only averaged values of $T^{4}$ and the absorption coefficient at several second intervals need be passed from FSI to ANSYS. It also opens the possibility of coupling the fire and thermal analyses together. So long as hundreds of FDS updates can be performed between solid phase thermal updates, the cost of restarting FDS with a corrected set of thermal boundary conditions derived from the solid phase analysis can be minimized. This issue will be addressed again below.

Predicted temperatures in the steel structural components of the 96th floor of the north tower 100 minutes after impact are shown in Fig. 6. This is one of a series of development simulations, and is not necessarily the best prediction of either the thermal or mechanical state of the structure at that time. However, it does illustrate the extent to which the FSI permits highly detailed calculations of the thermal state of the structure to be coupled with FDS simulations. The peak temperatures in this simulation reach $675^{\circ} \mathrm{C}$ near the south face of the floor, opposite the damaged portion of the floor. This is roughly the area reached by the fires several minutes earlier as they moved about the floor. The simulation includes the concrete slab and the insulation material, which are omitted for clarity.

The above simulation spans a range of length scales ranging from over $60 \mathrm{~m}$ (the tower width) to $1 \mathrm{~cm}$ (the radius of a truss rod). The entire floor system of each individual floor, including insulation and the concrete floor slab, is coupled together in a single calculation. The ANSYS model for a single combined floor system contains approximately 450,000 nodes and 650,000 elements [13]. Each column is simulated separately, since they are weakly coupled thermally. The column models have 20 nodes per floor in the vertical direction and a few hundred to describe the heat transfer in each horizontal cross section. Thus, the number of nodes and elements in the columns is comparable to that in the floor system. It takes approximately one day per floor to perform a thermal analysis. Clearly, 


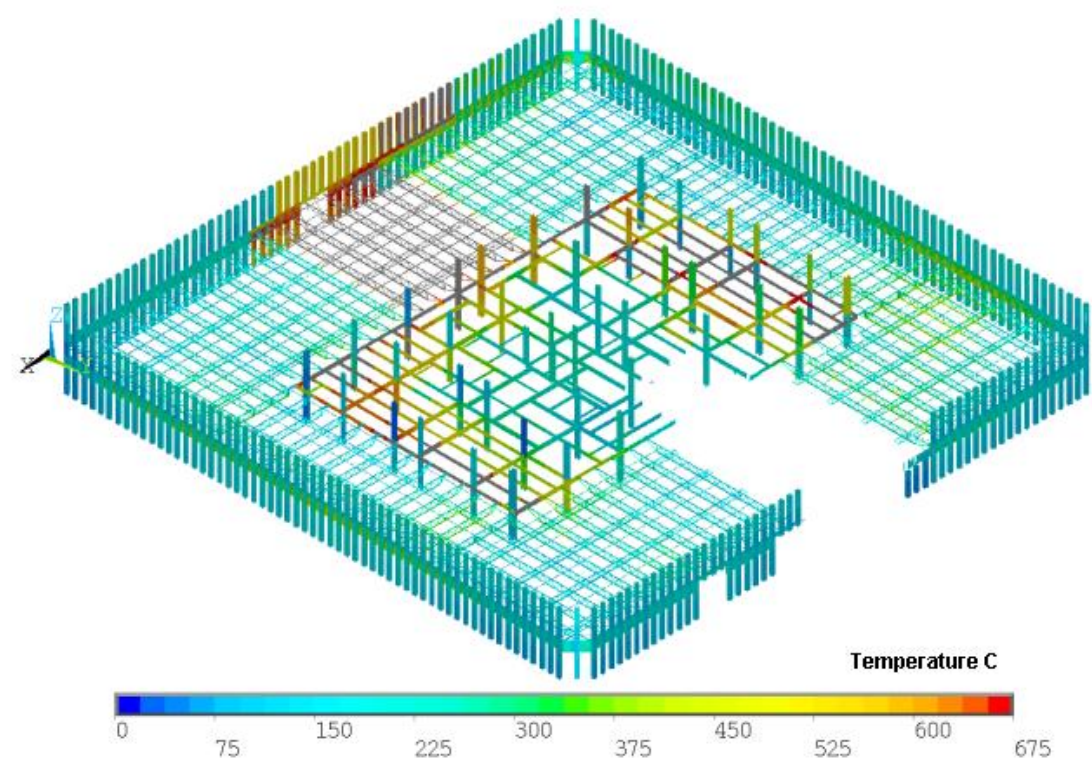

Fig. 6. Predicted temperatures in the steel structure of the 96th floor of the north tower 100 minutes after impact. The peak temperatures reach $675^{\circ} \mathrm{C}$. The calculation for each floor includes the insulation and concrete slab (not shown). Courtesy K. Prasad.

calculations at this level of detail are only possible because of the relative simplicity of conduction heat transfer compared with turbulent combustion. The fact that each floor can be simulated independently is itself a form of parallel processing. It requires many copies of the simulation code (ANSYS in this case), as opposed to a single copy of FDS for the fire simulations. However, financial issues aside, it leads to highly efficient computations.

The efficiency of the computations is of more than passing interest. Given the propagation of uncertainty through the chain of simulations, it is not possible to obtain credible results without many repetitions of each calculation. Only in this way can the sensitivity of the calculations to plausible variations in input be established. Moreover, the only way to make contact with the photographs and videos that provide a check on the credibility of the simulations is to repeat them enough times to establish the plausibility of the results. Tens of calculations of the type illustrated in the figures shown here have been performed as part of the NIST WTC investigation.

One novel feature of the thermal analysis performed by the FSI is worth special mention. The thickness of the spray-on insulation on the trusses in the WTC towers has been the focus of considerable attention [18]. However, given the method of application, the thickness can only be defined statistically, as there is a wide variation in the actual thickness at each point along the protected components. Figure 7 illustrates the methodology developed to deal with this fact. An artificially thick uniform insulation model is built up around each protected rod. Then a mean thickness and variance are chosen, and individual elements are 

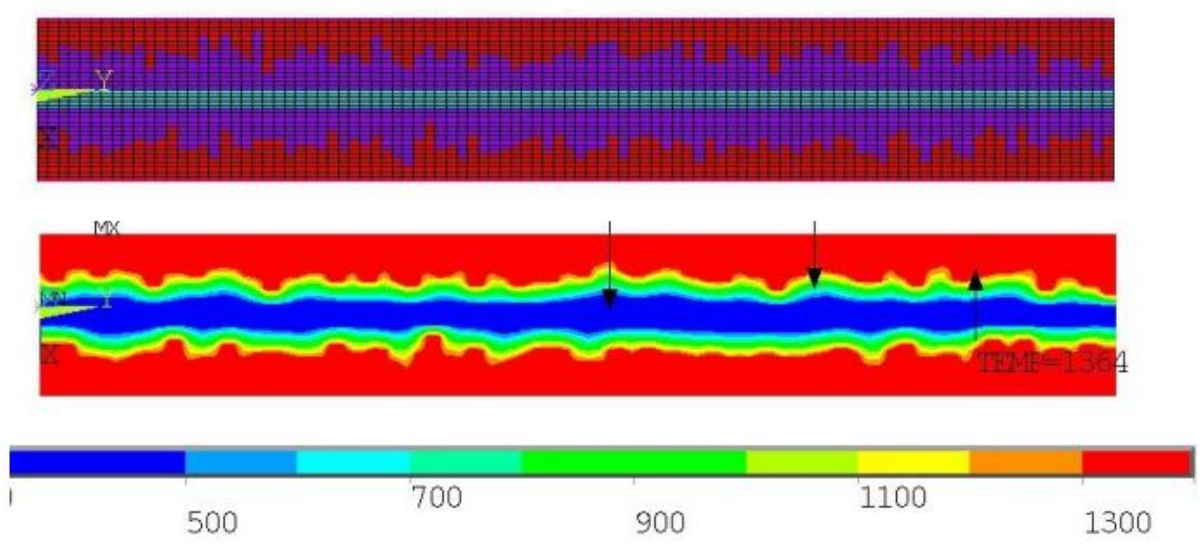

Fig. 7. Finite element model of spray-on insulation showing random variations in thickness (top). The outer layer of cells have no thermal inertia, the middle layer represents the actual insulation whose thickness is specified statistically, and the inner layer simulates the steel rod. The lower figure shows the computed temperatures. Courtesy K. Prasad.

chosen statistically to have either the proper thermal properties or essentially no thermal inertia. The result is a finite element model whose geometric simplicity is preserved but which accounts for the loss of protection associated with random thickness variations. The result is a net loss for a given average thickness because those portions of the rod with less protection experience higher heat transfer. The excess heat can be transferred a small distance along the rod by axial diffusion, canceling whatever reduction might have resulted from the thicker adjacent insulation. This also gives rise to local "hot spots", which can cause a weakening of the structural element in the immediate vicinity of the spot.

\section{RESEARCH ISSUES}

The remaining issues to be discussed here concern the coupling between the thermal analysis of the structure and the calculation of stress and displacement fields. While they may not be "research issues" in the strictest sense of the word, the extent to which these phenomena need to be coupled is not adequately appreciated in the view of this author. The first point worth emphasizing is that the spatial and temporal resolution of the stress analysis cannot be chosen arbitrarily once the requirements for thermal analysis have been decided.

Consider the idealized problem of a three-dimensional elastic half-space that is loaded thermally by a moving point source of heat $q_{o} Q_{T}(\tau)$ concentrated at a point on the free surface $\vec{r}=\vec{R}(\tau)$. Then, the classic theory of linear thermo-elasticity can be used to show that without approximation the surface temperature rise and the normal component of the surface displacement (the "bulge") take the following form (see Fig. 8):

$\Theta(\vec{r}, \tau)=\int_{0}^{\tau} d \tau_{o} Q_{T}\left(\tau_{o}\right) G\left(\tau-\tau_{o}, \eta_{T}\right) \quad \eta_{T}=\left|\vec{r}-\vec{R}\left(\tau_{o}\right)\right| / \sqrt{\left(\tau-\tau_{o}\right)}$ 


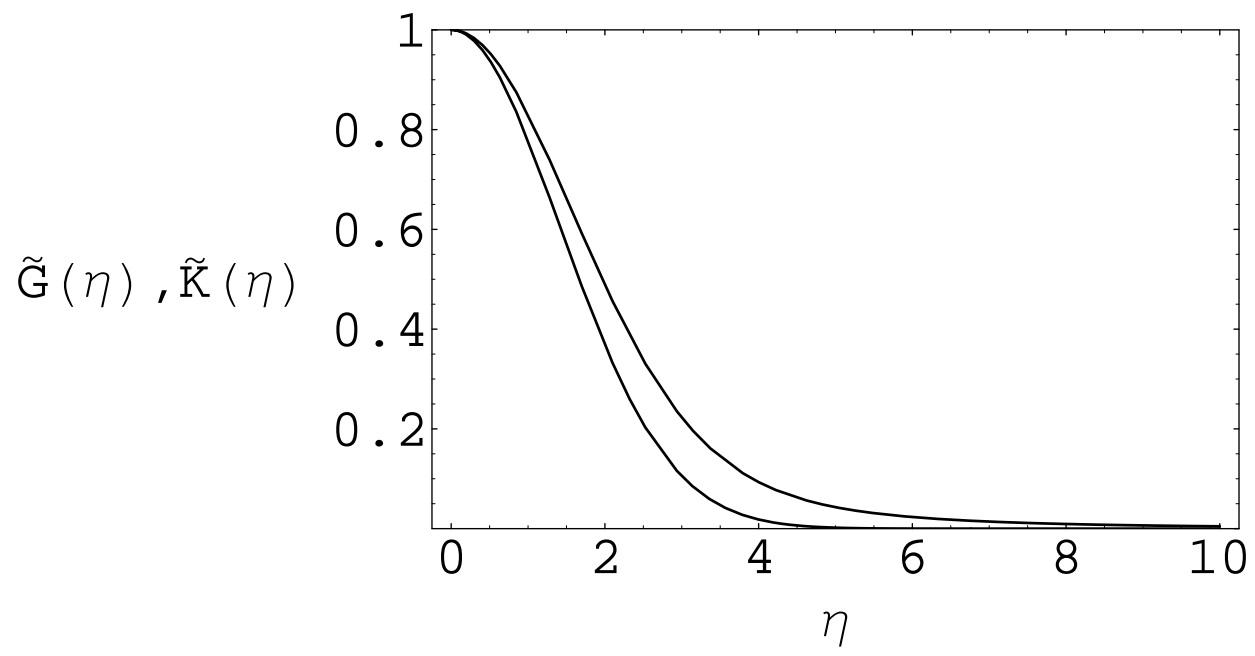

Fig. 8. Kernel functions normalized by their value at $\eta=0$. The quantities actually plotted are $\tilde{G}(\eta)=G(\eta, \tau) / G(0, \tau)$ and $\tilde{K}(\eta)=K(\eta, \tau) / K(0, \tau)$. The Gaussian function $G$ decays more rapidly than $K$ which decays $\sim \eta^{-3}$.

$w(\vec{r}, \tau)=\int_{0}^{\tau} d \tau_{o} Q_{T}\left(\tau_{o}\right) K\left(\tau-\tau_{o}, \eta_{T}\right) \quad \eta_{T}=\left|\vec{r}-\vec{R}\left(\tau_{o}\right)\right| / \sqrt{\left(\tau-\tau_{o}\right)}$

Here, $G$ is the classical Gaussian Kernel function and $K$ is an analogous function that emerges from the thermo-elastic analysis. The variable $\tau$ is a time normalized with the time scale $t_{o}$ imposed by the thermal heating, and the length scale $l=\sqrt{t_{o} / \alpha}$ where $\alpha$ is the thermal diffusivity. The dimensionless temperature rise $\Theta$ is made non-dimensional with respect to $q_{o} l / k$, where $k$ is the thermal conductivity. The surface displacement $w$ is normalized by $l$ and a thermo-elastic "coupling constant". The function $K$ can be written explicitly as:

$K=-\left(\frac{2 \mu+\lambda}{\mu+\lambda}\right) \frac{1}{8 \pi \tau} \exp \left(-\eta^{2} / 8\right)\left(I_{o}\left(\eta^{2} / 8\right)-I_{1}\left(\eta^{2} / 8\right)\right)$

The quantities $\lambda$ and $\mu$ are the Lamé constants of classical elasticity, while $I_{0}$ and $I_{1}$ are Modified Bessel functions. These results are probably well known, although I cannot find them in the classical literature,

The importance of these formulae lies in the fact that both the temperature rise and displacement are shown to be inherently time-dependent diffusive processes, with the time scale and diffusion set by the temperature field. Moreover, the displacements depend explicitly on the entire space-time history of the thermal heating of the exposed surface. If the temperature field is independent of time or if it is postulated as opposed to derived, then this issue does not arise. However, under either of these circumstances, it is no longer possible to couple the fire dynamics to the structural analysis. While the problems of interest in a structural collapse scenario are inherently non-linear, that does not invalidate the above 


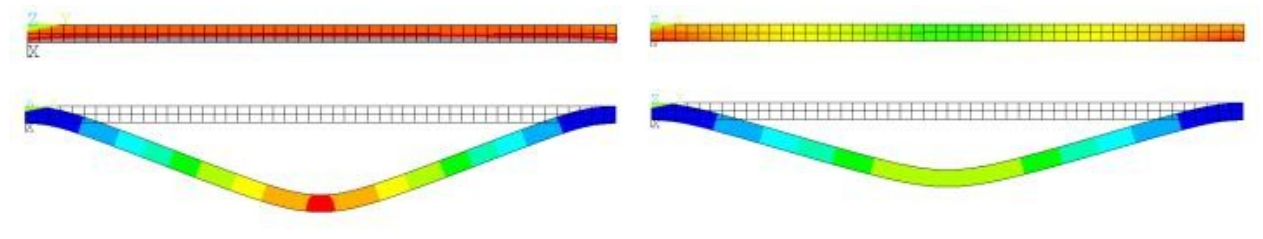

Fig. 9. Temperatures (top) and displacements of an unprotected steel rod in a gas with a vertically stratified temperature profile. The simulation on the left assumes the heat transfer to the rod is evaluated at its original position while the right hand simulation uses the current position of the rod. Courtesy K. Prasad.

conclusions. The thermally induced diffusion is then superimposed on a variety of other phenomena.

The significance of geometric non-linearity caused by large thermally induced displacements has recently been emphasized by Lane [19]. This has important consequences for coupled thermal-structural analyses. Even if large deflections in components of the structure do not change the fire dynamics appreciably, the deformation can change the thermal environment experienced by the component. Figure 9 shows a comparison between two time dependent ANSYS simulations of a horizontal rod fixed at the ends and placed in a gas with a temperature distribution that increases with increasing height. The simulation on the left assumes the heat transfer to the rod is evaluated at its original position while the right hand simulation uses the current position of the rod. The upper part of the figure shows the temperatures while the deflections are shown below. Note that the temperature in the left hand simulation is uniform, because the heat flux to the rod is always evaluated at the original location, even though the temperature in the rod increases with time. The change in heat flux to the rod with changing location is included in the right hand simulation. As a result, the rod temperature varies significantly with position. The deflections in this case are smaller, because as the rod deforms it moves into a cooler environment with lower heat transfer as a result. While this may not seem too important for an isolated truss rod, the consequences if repeated on a large scale can be quite significant [1], [19]. Both effects illustrated in this section could be addressed if thermal and stress analyses of a given structure were performed in parallel rather than sequentially. The difficulty arises in choosing a consistent level of modeling detail which preserves the ability to perform the stress analysis without compromising the accuracy of the temperature field.

\section{CONCLUDING REMARKS}

A discussion of the current state of our ability to predict the effects of fire on building structures has been presented. The emphasis has been on the degree to which fire dynamics, heat transfer, and structural analysis can be coupled. The NIST investigation into the collapse of the WTC towers is used as a backdrop, because it provides a concrete illustration of the strengths and limitations of our current methodology. While the simulation capability in each of these fields can (and no doubt will) be advanced separately, it is only when they are coupled together in some way that fire effects on structures can be quantitatively assessed. This is an area that would benefit greatly from increased attention by the Fire Research 
community. I hope this community will rise to the challenge.

\section{ACKNOWLEDGEMENTS}

It is a great honor for me to be able to give a lecture dedicated to the memory of Prof. Howard W. Emmons. He was a mentor to me for the entire duration of my stay at Harvard University, first as a graduate student and then as a junior faculty member. At NIST, I have been fortunate to be influenced by many gifted colleagues. My greatest debt is to my long time friend and collaborator Dr. Ronald Rehm. Dr. Kevin McGrattan and Dr. Kuldeep Prasad performed much of the work I have described here, and generously shared their results with me. There are many others both inside and outside NIST, too numerous to name here, who have also helped me over the years. I thank them all from the bottom of my heart.

Certain commercial software is identified in this paper to foster understanding. Such identification does not imply recommendation or endorsement by the National Institute of Standards and Technology, nor does it imply that the software identified is necessarily the best available for the purpose.

\section{REFERENCES}

[1] A.S. Usmani, Y.C. Chung, and J.L. Torero. How did the WTC towers collapse: a new theory. Fire Safety Journal, 38: 501-534, 2003.

[2] A. Hori, Analytical Method for High Temperature Collapse of a 3D Steel Frame. Fire Science and Technology, 23: 208-221, 2004.

[3] N. Abboud, M. Levy, D. Tennant, J. Mould, H. levine, S. King, C. Ekwueme, A., Jain, and G. Hart. Anatomy of the World Trade Center collapses: a structural engineering investigation, SEI/SFPE Conference on Designing Structures for Fire, Baltimore, MD, September 2003.

[4] C. Beyler, D. White, M. Peatross, J. Trellis, S. Li, A. Luers, and D. Hopkins. Analysis of Fire Development in the WTC Attacks. SEI/SFPE Conference on Designing Structures for Fire, Baltimore, MD, September 2003.

[5] G. Thater, G. Panariello, and D. Cuoco. World Trade Center Disaster: Damage/Debris Assessment. ASCE 3rd Forensics Congress. San Diego, CA, October 2003.

[6] J.O. Hallquist. LS-DYNA Theoretical Manual, Livermore Software Technology Corporation, Livermore, CA, May 1998.

[7] D.K. Vaughan, FLEX Users Guide, Report UG8298, Weidlinger Associates Inc., Los Altos, CA, May 1983 with updates through 2002.

[8] K.B. McGrattan (Editor) Fire Dynamics Simulator (Version 4), Technical Reference Guide. NIST Special Publication 1018, National Institute of Standards and Technology, Gaithersburg, MD, July 2004.

[9] K.B. McGrattan and C. Bouldin. Simulating the fires in the World Trade Center. Interflam 2004, proceedings of the tenth International Conference, Interscience, London, 999-108, 2004. 
[10] R.G. Rehm, W.M. Pitts, H.R. Baum, D.D. Evans, K. Prasad, K.B. McGrattan, and G.P. Forney. Initial Model for Fires in the World Trade Center. NISTIR 6879, National Institute of Standards and Technology, Gaithersburg, MD, May 2002.

[11] H.R. Baum and R.G. Rehm. A Simple model of the World Trade Center fireball dynamics, Proceedings of the Combustion Institute, 30: 2247-2254, 2005.

[12] ANSYS Release 8.0 Documentation, ANSYS Corp., 2003.

[13] K. Prasad and H.R. Baum. Coupled fire dynamics and thermal response of complex building structures. Proceedings of the Combustion Institute, 30:2255-2262, 2005.

[14] Y. Hasemi. Diffusion Flame Modeling as a Basis for the Rational Fire Safety Design of Built Environments. Fire Safety Science - Proceedings of the Sixth International Symposium, M. Curtat, Ed., International Association for Fire Safety Science. 3-22, 2000.

[15] A. Hamins, K. McGrattan, K. Prasad, A. Maranghides, and T. McAllister. Experiments and Modeling of Unprotected Structural Steel Elements Exposed to Fire, Fire Safety Science - Proceedings of the Eighth International Symposium, in press, 2005.

[16] P. Pagni. Thermal Glass Breakage. Fire Safety Science - Proceedings of the Seventh International Symposium, D.D.Evans, Ed., International Association for Fire Safety Science. 3-22, 2003.

[17] Siegel, R. and Howell, J.R., Thermal Radiation Heat Transfer, Third Ed., Hemisphere, Washington, D.C., 699-722, 1992.

[18] J.G. Quintiere, M. di Marzo, and R. Becker, A Suggested Cause of the fire-induced collapse of the World Trade Towers, Fire Safety Journal 37: 707-717, 2002.

[19] B. Lane. A Suggested Cause of the fire-induced collapse of the World Trade Towers, Fire Safety Journal 38: 589-591, 2003. 